\title{
Differential Efficacy of Two Dental Implant Decontamination Techniques in Reducing Microbial Biofilm and Re-Growth onto Titanium Disks In Vitro
}

\author{
Aida Meto ${ }^{1,+}\left(\mathbb{D}\right.$, Enrico Conserva ${ }^{2,+}\left(\mathbb{D}\right.$, Francesco Liccardi $^{2}$, Bruna Colombari ${ }^{3}$, Ugo Consolo ${ }^{2} \mathbb{C}$ \\ and Elisabetta Blasi ${ }^{3, *}$ \\ 1 School of Doctorate in Clinical and Experimental Medicine, Laboratory of Microbiology and Virology, \\ University of Modena and Reggio Emilia, 41125 Modena, Italy \\ 2 Department of Surgery, Medicine, Dentistry and Morphological Sciences with interest in Transplant, \\ Oncology and Regenerative Medicine, Operative Unit of Dentistry and Maxillofacial Surgery, \\ University-Hospital of Modena, 41125 Modena, Italy \\ 3 Department of Surgery, Medicine, Dentistry and Morphological Sciences with interest in Transplant, \\ Oncology and Regenerative Medicine, Laboratory of Microbiology and Virology, 41125 Modena, Italy \\ * Correspondence: elisabetta.blasi@unimore.it; Tel.: +39-592-055-468 \\ + These authors contributed equally to this work.
}

Received: 19 July 2019; Accepted: 2 August 2019; Published: 5 August 2019

\begin{abstract}
Dental implants are crucial therapeutic devices for successful substitution of missing teeth. Failure cases are mainly pathogen-associated events, allowing clinical progression toward peri-mucositis or peri-implantitis. The aim of this study was to compare the performance of two mechanical decontamination systems, Nickel-Titanium brush (Brush) and Air-Polishing system with $40 \mu \mathrm{m}$ bicarbonate powder (BIC-40), by means of a novel bioluminescence-based model that measures microbial load in real time. Briefly, 30 disks were contaminated using the bioluminescent Pseudomonas aeruginosa strain (BLI-P. aeruginosa), treated with Brush (30 s rounds, for $90 \mathrm{~s}$ ) or BIC-40 (30 s, at $5 \mathrm{~mm}$ distance) procedure, and then assessed for microbial load, particularly, biofilm removal and re-growth. Our results showed that Brush and BIC-40 treatment reduced microbial load of about 1 and more than 3 logs, respectively. Furthermore, microbial re-growth onto Brush-treated disks rapidly occurred, while BIC-40-treated disks were slowly recolonized, reaching levels of microbial load consistently below those observed with the controls. In conclusion, we provide evidence on the good performance of BIC-40 as titanium device-decontamination system, the clinical implication for such findings will be discussed.
\end{abstract}

Keywords: Brush; BIC-40; BLI-P. aeruginosa; dental implants; decontamination systems; titanium disks

\section{Introduction}

To achieve a successful oral rehabilitation, aimed at substituting one or more missing teeth via dental implants, such as titanium (Ti) element or titanium alloys [1], it is important not only to obtain implant osseointegration but also to limit, as much as possible, the risk of peri-implant disease [2]. As recently underlined [3], much has to be done in such topic. Peri-implantitis is a chronic, irreversible, multifactorial condition, triggered by microbial biofilm formation, and persistent inflammation around dental implants [3-5], especially in patients with insufficiently poor oral hygiene, smoking, diabetes, history of periodontitis, trauma, or fracture due to uncontrolled implant-overload [6,7]. Massive and long-lasting inflammation of the surrounding area will bring to local tissue de-structuration, bone-degradation and eventually loss of the involved implant(s) [8]. It has also been hypothesized that some subjects are genetically predisposed to the onset and progression of peri-implantitis. This would 
be due to an incorrect modulation of the RANK/RANKL systems, regulators of immune responses, and bone physiology [9-13]. In particular, three molecules, the receptor activator of NF-kappa B (RANK), its ligand RANKL, and the decoy receptor of RANKL, osteoprotegerin (OPG), attracted the attention of scientists and pharmaceutical companies alike. Genetic experiments evolving around these molecules established their pivotal role as central regulators of osteoclast function [14].

Notoriously, microbial contamination and biofilm formation may take place onto biotic and abiotic surfaces, including dental implants. Once established as a sessile structure, microbial community acquires enhanced resistance to cleansing techniques, antimicrobial drugs, and host immune defenses with respect to their planktonic counterpart $[15,16]$. Several studies $[17,18]$ focused on implant and abutment colonization, stating that the characteristics of their surface(s) significantly influence clinical outcome, affecting microbial localization on the device(s) and around the peri-implant area. Moreover, by an experimental study in dogs, Albouy et al. [19] demonstrated that peri-implantitis progression is more rapid when using rough surface implants rather than machined ones.

Over the years, different methods, such as mechanical and chemical systems or antimicrobial solutions, have been used for implant decontamination, thus achieving good results [20,21]. Nevertheless, undesired alteration(s) of implant surfaces can happen to a different extent [22]. Current efforts are aimed to implement microbial biofilm removal, minimizing changes/damages on implant surface-structure(s) and, at meantime, maintaining the implant biologically adequate and favorable for adhesion and persistence of reparative host cells [23,24].

Among several decontamination systems, Nickel Titanium brushes (Brush) and Air Polishing system with $40 \mu \mathrm{m}$ bicarbonate powder (BIC-40) are receiving interesting attention. The former is known to be resistant and high flexible material when undergoing heating/cooling variations [25]. The latter, employing bicarbonate powder, is often used by clinicians for its efficacy in mechanical removal of biofilm, providing satisfactory clinical outcomes [26].

The set-up of new experimental models, such as the recently described use of an engineered laboratory strain of Pseudomonas aeruginosa (P. aeruginosa) to assess, in real time, biofilm formation onto medical devices [27], opens new pathways for a better comprehension of the events involved in microbial biofilm formation onto abiotic surfaces and its abatement by chemical and/or mechanical procedures.

The aim of the present study was to compare two decontamination systems, Brush and BIC-40, in their ability to impair P. aeruginosa contamination onto titanium disks by a bioluminescence-based assay. The null hypothesis was that biofilm persistence and microbial re-growth on titanium disks were comparable, irrespectively of the decontamination system employed.

\section{Materials and Methods}

\subsection{Titanium Disks}

We tested 30 disks (MegaGen Co. Ltd., Daegu, South Korea), with Calcium-incorporated titanium surface, having dimensions of $13 \mathrm{~mm}$ in diameter, $3 \mathrm{~mm}$ in thickness, and an arithmetic average (Ra) value of $0.93 \mu$. The titanium disks were firstly sterilized by autoclave. Then, microbial contamination and cleaning/decontamination were performed as below.

\subsection{Pseudomonas Aeruginosa}

We used the bioluminescent P. aeruginosa strain P1242 (BLI-Pseudomonas). As previously described [28], such cells were engineered in order to express the luciferase gene and its substrate under the control of a constitutive P1 integron promoter 2, thus constitutively producing a bioluminescent signal. Bacteria from $-80^{\circ} \mathrm{C}$ glycerol stocks were initially seeded onto Tryptic Soy Agar (TSA) (OXOID, Milan, Italy) plates and incubated overnight at $37^{\circ} \mathrm{C}$. Then, isolated colonies were collected, suspended with $10 \mathrm{~mL}$ of Tryptic Soy Broth (TSB) (OXOID, Milan, Italy), and allowed to grow overnight at $37^{\circ} \mathrm{C}$ with gentle shaking. Bacterial concentrations were then assessed by the McFarland standard 
curve and working dilutions $\left(1 \times 10^{6} / \mathrm{mL}\right.$, in TSB) were prepared and used according to the protocols detailed below.

\subsection{Microbial Growth and Biofilm Formation onto Titanium Disks}

Overnight cultures of BLI-Pseudomonas $\left(10^{6} / \mathrm{mL}\right)$ were seeded in 96 black well-plates, in the presence or not of titanium disks (1 disk/well), to allow microbial growth and biofilm formation. The plates were then incubated at $35^{\circ} \mathrm{C}$ for $24 \mathrm{~h}$, into the Fluoroskan Luminescence reader (Thermo Fisher Scientific, Waltham, MA, USA), and the bioluminescence was detected hourly. The BLI signal was automatically recorded (total microbial load). At time $24 \mathrm{~h}$, the disks were washed twice with phosphate buffered saline (PBS) (EuroClone, Wetherby, UK) at room temperature (RT) to eliminate the planktonic cells, transferred into new wells and the bioluminescence signal was again measured (biofilm produced onto disk surfaces). The recorded bioluminescence values were expressed as Relative Luminescence Units/sec (RLU/sec) and converted in colony forming units (CFU/disk), based on an internal reference curve.

\subsection{Titanium Disk Decontamination Systems}

BLI-Pseudomonas contaminated disks were treated with Nickel-Titanium Brushes, named Brush (Implant Cleaning Technique, Hans Korea Co. Ltd., Gyeonggi-do, Korea/De Ore, Verona, Italy) and Air-Polishing system (Combi-Touch, Mectron spa, Carasco, Genova, Italy) with $40 \mu \mathrm{m}$ bicarbonate powder (BIC-40). The Brush, composed of about 40 super elastic filaments with a diameter of $0.07-0.13 \mathrm{~mm}$, was used at 400 and $600 \mathrm{rpm}$, for three sequential rounds of $30 \mathrm{~s}$ each with $25 \mathrm{~g}$ pressure calibrated on an electronic scale of $100 \mathrm{~N}$ torque. The BIC-40 system ( $30 \mathrm{~s}$ at a distance of $5 \mathrm{~mm}$ ) consisted in the mechanical action of compressed air spreading a flow of particles onto the titanium surface, as previously demonstrated by the authors [25]. The BIC-40 system was executed for $30 \mathrm{~s}$ at a distance of $5 \mathrm{~mm}$, consisting in the mechanical action of compressed air spreading the flow of particles onto the titanium surface. When the particles hit the surface, their kinetic energy is dissipated almost completely, thus producing a gentle and effective cleansing action. The cleaning treatment was completed by a water jet that was arranged in the form of a bell around the main flow. This flow used the pressure drop originated around the nozzle to prevent the powder cloud from bouncing and being dispelled and, at the same time, to dissolve the powder by washing the surface.

All the decontamination treatments were performed by the same operator.

\subsection{Residual Biofilm and Microbial Re-Growth after Decontamination}

The BLI-Pseudomonas-contaminated titanium disks were divided into three groups and processed as: untreated controls, Brush-treated, and BIC-40-treated groups. Upon treatment, the disks were transferred in new wells containing fresh medium and immediately analyzed by the Fluoroskan reader (residual biofilm). Subsequently, the disks were incubated at $35{ }^{\circ} \mathrm{C}$ into the Fluoroskan reader and hourly evaluated for BLI signal for further $24 \mathrm{~h}$ (microbial regrowth). The values obtained were expressed as RLU/sec and CFU/disk.

\subsection{Statistical Analysis}

Each experiment was performed twice; each protocol included 15 disks. Each condition (i.e., control, Brush and BIC-40) was tested using five replicates. Data that had a normal distribution were expressed as RLU/sec or CFU/disk. The results are shown as mean \pm standard error (SEM). Statistical analysis was performed using the Student's $t$-test that allowed to compare treated vs untreated groups. 


\section{Results}

\subsection{Total Microbial Growth and Biofilm Formation onto Titanium Disks}

Initially, to exclude any effect of titanium on total microbial growth, BLI-P. aeruginosa $\left(10^{6} / \mathrm{mL}\right)$ was seeded at time 0 in two sets of wells, containing or not titanium disks ( 1 disk/well). Then, the plate was incubated at $35^{\circ} \mathrm{C}$ for $24 \mathrm{~h}$, and microbial growth was kinetically assessed, by bioluminescence analysis. Super-imposable and time-related trends were observed in the two groups (both above $10^{8}$ $\mathrm{CFU} / \mathrm{mL}$, at time $24 \mathrm{~h}$ ), indicating that the presence of titanium disks did not affect P. aeruginosa growth. Next, the formation of $P$. aeruginosa biofilm onto titanium disks was assessed. In particular, at $24 \mathrm{~h}$, the disks were washed twice with PBS to eliminate the non-adherent microbial cells, transferred into new wells, and then tested for residual bioluminescent signal. As shown in Figure 1 (upper panel, control group), BLI-RLU remained at levels as high as $0.8 \pm 0.12 \mathrm{RLU} / \mathrm{s}$, indicating that a strong biofilm had been formed onto the disks. In order to express the BLI results as microbial load/disk, an internal calibration curve was used, and the CFU/disk were calculated, as shown in Figure 1 (lower panel, control group).

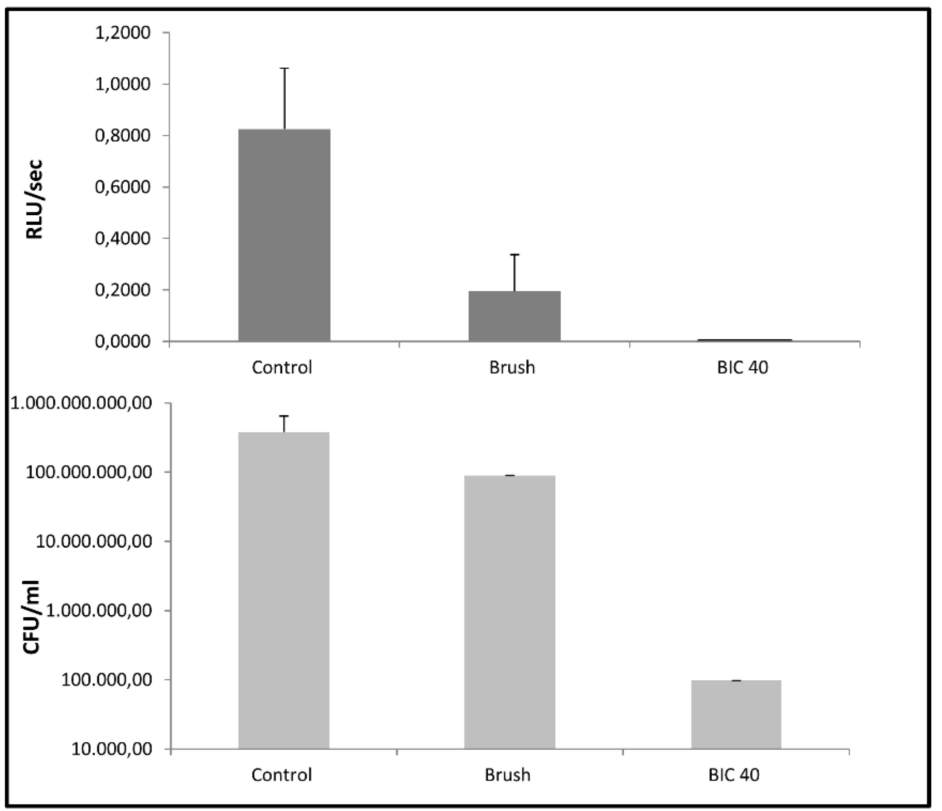

Figure 1. P. aeruginosa biofilm formation onto Titanium disks and residual microbial load, following $\mathrm{Ni}$-Ti brush and AirPol BIC-40 decontamination treatments. Microbial load was assessed on treated and untreated disks, by bioluminescence assay; the results are given as RLU/s and number of viable cells (CFU/disk).

\subsection{Microbial Biofilm Removal from Titanium Disks by the Two Decontamination Systems}

To assess the efficacy of the two cleaning systems, two sets of disks, housing a 24 h-old P. aeruginosa biofilm, were treated with Brush or BIC-40 procedures, as detailed in Section 2. In parallel, a third set of disks remained untreated (control group). Then, each disk was transferred into a new well, with fresh medium, and the RLU/s were immediately detected. As shown in Figure 1, when compared to the untreated control group, both decontamination systems were capable of impairing biofilm level, although to a different extent. In particular, when expressing such decreases as percent, the Brush procedure caused $76 \%$ biofilm reduction, while the BIC-40 treatment allowed $99.9 \%$ of biofilm reduction.

\subsection{Microbial Re-Growth onto Treated Titanium Disks}

The microbial re-growth onto decontaminated titanium disks was measured hourly, for additional $24 \mathrm{~h}$, incubating again the plate at $35^{\circ} \mathrm{C}$ in the Fluoroskan reader. The results are given as microbial 
load ratio between treated and untreated disks, as shown in Figure 2. We found that, following the Brush procedure, the microbial load ratios rapidly increased, up to about 1.5 logs within the initial $5 \mathrm{~h}$. Then, between treated and untreated groups, values remained constantly close to 1 up to $24 \mathrm{~h}$, indicating that microbial load in treated and untreated disks was comparable.

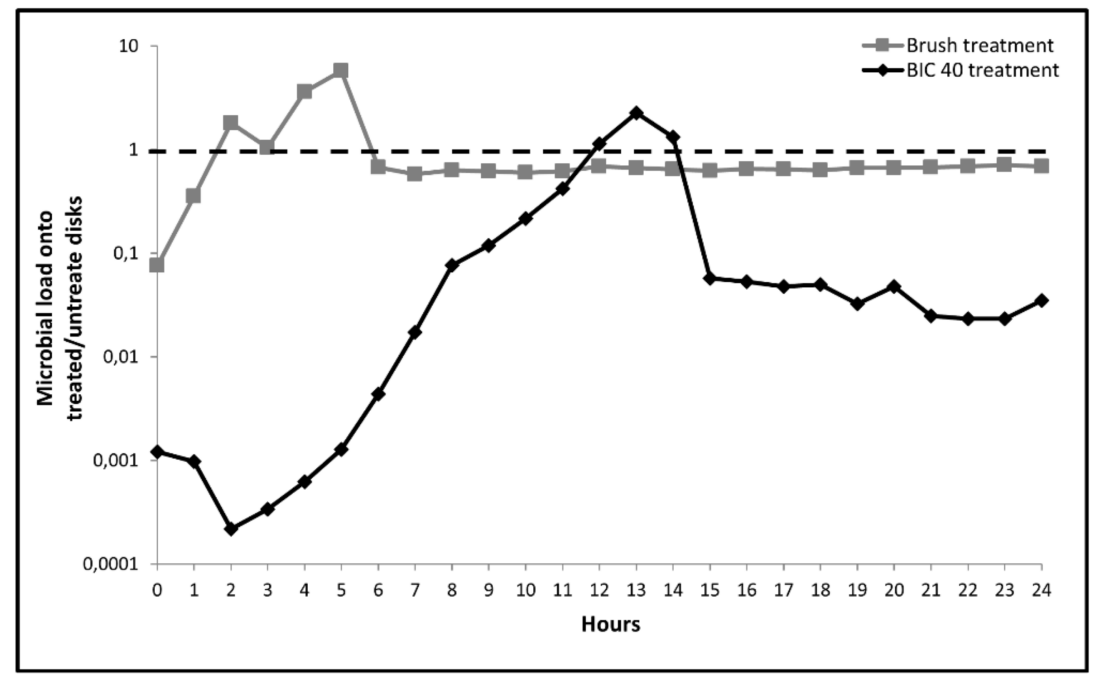

Figure 2. Kinetic evaluation of $P$. aeruginosa biofilm re-growth onto titanium disks, following decontamination by Ni-Ti Brush and AirPol BIC-40 systems. Microbial load was kinetically assessed on treated and untreated disks, by bioluminescence assay. The results are expressed as microbial load ratio between treated and untreated disks. The ratio $=1$ (dotted line) indicates superimposable microbial load in treated and untreated groups.

Differently, when using the BIC-40 procedure, the microbial load ratios further decreased at time 2-4 hours of incubation. Then, a gradual but slow enhancement was observed up to 12-13 hours. After that, the values decreased again and remained stable by the time, the ratio between treated and untreated disks ranging between 0.1 and $0.01 \mathrm{log}$. These data indicated that BIC-40-treated disks maintained, by the time, a significantly lower microbial load with respect to both the Brush-treated as well as the untreated control counterpart.

\section{Discussion}

Here, we show that BIC-40 system performs better than Brush in reducing P. aeruginosa biofilm and microbial re-growth onto titanium disks.

Dental implants are widely used in different clinical situations; osseointegration ability and long-lasting integrity bring about successful results [29]. Vice versa, microbial localization on the implant affects the surrounding area, eventually leading to osseointegration loss [3,4]. As described in [30], microbial biofilm may happen, despite titanium disks' surface sharpness. This outline needs a targeted clinical action for patient benefit. Besides antibiotics or other therapies [31], decontamination techniques of dental implant surfaces deserve special attention as local cleaning systems. Such treatments seem to be less effective when applied to rough surfaces rather than to smooth surfaces. Furthermore, depending upon cleaning procedure used, undesirable effects may occur on implant surface morphology [32-34].

By a recently established model assessing in real time microbial biofilm formation onto medical devices [27], here we show that BLI-Pseudomonas has the ability to adhere onto the titanium disks, thus rapidly forming a consistent biofilm on their surfaces within $24 \mathrm{~h}$. Moreover, we provide the first evidence on the different efficacy of the two decontamination treatments, since Brush and BIC-40 are capable of impairing biofilm up to $76 \%$ and $99.9 \%$, respectively. In particular, as evaluated by RLU analysis and subsequent conversion in CFU/disk, we show that microbial load is reduced of about $1 \log$ 
following Brush treatment, while more than 3 logs decrease occurs upon BIC-40 treatment. Furthermore, kinetic assessment of BLI signal demonstrates that microbial re-growth onto BIC-40-treated disks is delayed and remains mostly below the control values (about $1 \mathrm{log}$ ). In the Brush-treated disks, microbial re-growth occurs, rapidly reaching levels similar to those observed in the untreated control disks. It should be noted that Pseudomonas produces one of the most complex and difficult to treat biofilms [35] even on dental implants [36]. Thus, we assume that the successful decontamination obtained by BIC-40, in our model, would likely be as effective when applied against other clinically relevant biofilms, including those produced during peri-mucositis/peri-implantitis.

As recently published [25], a mechanical treatment for decontamination of titanium disks, such as the use of Brush, deeply affects the device surface producing deep grooves on it. In contrast, lack of damage occurs in BIC-40 treated titanium surface(s). Taken together, our previous [25] and present data favor the conclusion that the use of BIC-40, avoiding formation of niches onto device surface, would in turn prevent/minimize microbial adhesion, survival, and persistence. Moreover, we favor the hypothesis that the good performance of BIC- 40 can also be related to the fact that sodium bicarbonate particles, remaining onto BIC-40-treated titanium disks [25], likely render the device surface hostile to subsequent microbial re-growth. In any case, using a BLI-based system that allows kinetic monitoring of microbial load onto medical devices [27], we have been able to show a different efficacy of the two decontamination procedures not only in impairing preformed biofilm but also in limiting subsequent re-growth onto titanium disks.

Finally, it is worth noting that the present data have been obtained using titanium disks with rough surfaces, as representative of the worst condition clinically possible. Indeed, previous studies investigating the correlation between implant surface morphologies and microbial localization around the peri-implant areas demonstrate a more pronounced progression of the pathology around rough surface rather than machined ones [17-19]. From here, we assume that BIC-40 performance would further improve when used to decontaminate machinated implants.

\section{Conclusions}

This in vitro study provides evidence on the relevance of BIC-40 as dental implant decontamination system. Besides avoiding titanium surface damage [25], such treatment consistently impairs biofilm and microbial re-growth. Given the relevance of microbial contamination in implant failure, these findings open to a clinical use of BIC-40 in the treatment of pathogen-related peri-mucositis and peri-implantitis.

Author Contributions: Conceptualization, E.B., E.C., and F.L.; Methodology, A.M., F.L., and B.C.; Software, A.M., B.C., F.L., and E.C.; Validation, E.C., A.M., and E.B.; Formal analysis, B.C.; Investigation, F.L.; Resources, U.C.; Data curation, U.C. and E.B.; Writing-original draft preparation, A.M. and E.C.; Writing-review and editing, E.B. and U.C; Visualization, B.C. and F.L; Supervision, A.M., B.C., and E.B.; Project administration, E.C., and F.L.; Funding acquisition, U.C.

Funding: The work was partially supported by Mectron spa, Carasco, Genova, Italy (U.C.).

Acknowledgments: The Authors thank Dr. Eva Pericolini, University of Modena and Reggio Emilia, Modena, Italy, for kindly providing the BLI-Pseudomonas strain and for the methodological support in order to use such bacteria in our model.

Conflicts of Interest: The authors declare no conflict of interest.

\section{References}

1. Esposito, M.; Ardebili, Y.; Worthington, H.V. Interventions for replacing missing teeth: different types of dental implants. Cochrane Database Syst. Rev. 2015. [CrossRef]

2. Singh, P. Understanding peri-implantitis: a strategic review. J. Oral Implants 2011, 37, 622-626. [CrossRef] [PubMed]

3. Matarese, G.; Ramaglia, L.; Fiorillo, L.; Cervino, G.; Lauritano, F.; Isola, G. Implantology and Periodontal Disease: The Panacea to Problem Solving? Open Dent. J. 2017, 11, 460-465. [CrossRef] [PubMed] 
4. Botero, J.E.; González, A.M.; Mercado, R.A.; Olave, G.; Contreras, A. Subgingival microbiota in peri-implant mucosa lesions and adjacent teeth in partially edentulous patients. J. Periodontol. 2005, 76, 1490-1495. [CrossRef] [PubMed]

5. Schwarz, F.; Derks, J.; Monje, A.; Wang, H.L. Peri-implantitis. J. Periodontol. 2018, 89, S267-CS290. [CrossRef]

6. Klinge, B.; Flemming, T.; Cosyn, J.; De Bruyn, H.; Eisner, B.M.; Hultin, M.; Isidor, F.; Lang, N.P.; Lund, B.; Meyle, J.; et al. The patient undergoing implant therapy. Summary and consensus statements. Clin. Oral Implants Res. 2015, 26, 64-67. [CrossRef]

7. Mellonig, J.T.; Griffiths, G.; Mayths, E.; Spitznagel, J. Treatment of the failing implant: case reports. Int. J. Periodont. Rest. Dent. 1995, 15, 384-395.

8. Pye, A.D.; Lockhart, D.E.A.; Dawson, M.P.; Murray, C.A.; Smith, A.J. A review of dental implants and infection. J. Hosp. Infect. 2009, 72, 104-110. [CrossRef]

9. Leibbrandt, A.; Penninger, J.M. RANKL/RANK as key factors for osteoclast development and bone loss in arthropathies. Adv. Exp. Med. Biol. 2009, 649, 100-113.

10. Mizoguchi, T.; Muto, A.; Udagawa, N.; Arai, A.; Yamashita, T.; Hosoya, A.; Ninomiya, T.; Nakamura, H.; Yamamoto, Y.; Kinugawa, S.; et al. Identification of cell cycle-arrested quiescent osteoclast precursors in vivo. Cell Biol. 2009, 184, 541-554. [CrossRef]

11. Leibbrandt, A.; Penninger, J.M. RANK/RANKL: Regulators of immune responses and bone physiology. Ann. NY Acad. Sci. 2008, 1143, 123-150. [CrossRef] [PubMed]

12. Maruyama, K.; Takada, Y.; Ray, N.; Kishimoto, Y.; Penninger, J.M.; Yasuda, H.; Matsuo, K. Receptor activator of NF-kappa B ligand and osteoprotegerin regulate proinflammatory cytokine production in mice. J. Immunol. 2006, 177, 3799-3805. [CrossRef] [PubMed]

13. Wada, T.; Nakashima, T.; Hiroshi, N.; Penninger, J.M. RANKL-RANK signaling in Osteoclastogenesis and bone disease. Trends Mol. Med. 2006, 12, 17-25. [CrossRef] [PubMed]

14. Leibbrandt, A.; Penninger, J.M. RANK(L) as a key target for controlling bone loss. Adv. Exp. Med. Biol. 2009, 647, 130-145. [PubMed]

15. Meto, A.; Colombari, B.; Sala, A.; Pericolini, E.; Meto, A.; Peppoloni, S.; Blasi, E. Antimicrobial and antibiofilm efficacy of a copper/calcium hydroxide-based endodontic paste against Staphylococcus aureus, Pseudomonas aeruginosa and Candida albicans. Dent. Mater. J. 2019, 38, 591-603. [CrossRef]

16. Paulone, S.; Malavasi, G.; Ardizzoni, A.; Orsi, C.F.; Peppoloni, S.; Neglia, R.G.; Blasi, E. Candida albicans survival, growth and biofilm formation are differently affected by mouthwashes: An in vitro study. New Microbiol. 2017, 40, 45-52. [PubMed]

17. Subramani, K.; Jung, R.E.; Molenberg, A.; Hammerle, C.H. Biofilm on dental implants: a review of the literature. Int. J. Oral Maxillofac. Implants 2009, 24, 616-626.

18. De Freitas, A.R.; Silva, T.S.O.; Ribeiro, R.F.; de Albuquerque Junior, R.F.; Pedrazzi, V.; do Nascimento, C. Oral bacterial colonization on dental implants restored with titanium or zirconia abutments: 6-month follow-up. Clin. Oral Investig. 2018, 22, 2335-2343. [CrossRef]

19. Albouy, J.P.; Abrahamsson, I.; Berglundh, T. Spontaneous progression of experimental peri-implantitis at implants with different surface characteristics: An experimental study in dogs. J. Clin. Periodontol. 2012, 39, 182-187. [CrossRef]

20. Dennison, D.K.; Huerzeler, M.B.; Quinones, C.; Caffesse, R.G. Contaminated Implant Surfaces: An In Vitro Comparison of Implant Surface Coating and Treatment Modalities for Decontamination. J. Periodontol. 1994, 65, 942-948. [CrossRef]

21. Sahrmann, P.; Ronay, V.; Hofer, D.; Attin, T.; Jung, R.E.; Schmidlin, P.R. In vitro cleaning potential of three different implant debridement methods. Clin. Oral Implant. Res. 2015, 26, 314-319. [CrossRef]

22. Louropoulou, A.; Slot, D.E.; Van der Weijden, F. Influence of mechanical instruments on the biocompatibility of titanium dental implants surfaces: A systematic review. Clin. Oral Implant. Res. 2015, 26, 841-850. [CrossRef]

23. Mavrogenis, A.F.; Dimitriou, R.; Parvizi, J.; Babis, G.C. Biology of implant osseointegration. J. Musculoskelet. Neuronal Interact. 2009, 9, 61-71.

24. Wennerberg, A.; Albrektsson, T. Effects of titanium surface topography on bone integration: A systematic review. Clin. Oral Implant. Res. 2009, 20, 172-184. [CrossRef] 
25. Conserva, E.; Pisciotta, A.; Bertoni, L.; Bertani, G.; Meto, A.; Colombari, B.; Blasi, E.; Bellini, P.; de Pol, A.; Consolo, U.; et al. Evaluation of Biological Response of STRO-1/c-Kit Enriched Human Dental Pulp Stem Cells to Titanium Surfaces Treated with Two Different Cleaning Systems. Int. J. Mol. Sci. 2019, 20, 1868. [CrossRef]

26. Nemer Vieira, L.F.; Lopes de Chaves e Mello Dias, E.C.; Cardoso, E.S.; Machado, S.J.; Pereira da Silva, C.; Vidigal, G.M. Effectiveness of implant surface decontamination using a high-pressure sodium bicarbonate protocol: An in vitro study. Implant Dent. 2012, 21, 390-393. [CrossRef]

27. Pericolini, E.; Colombari, B.; Ferretti, G.; Iseppi, R.; Ardizzoni, A.; Girardis, M.; Sala, A.; Peppoloni, S.; Blasi, E. Real-time monitoring of Pseudomonas aeruginosa biofilm formation on endotracheal tubes in vitro. BMC Microbiol. 2018, 18, 84. [CrossRef]

28. Choi, K.H.; Schweizer, H.P. mini-Tn7 insertion in bacteria with single attTn7 sites: Example Pseudomonas aeruginosa. Nat. Protoc. 2006, 1, 153-161. [CrossRef]

29. Albrektsson, T.; Dahlin, C.; Jemt, T.; Sennerby, L.; Turri, A.; Wennerberg, A. Is marginal bone loss around oral implants the result of a provoked foreign body reaction? Clin. Implant Dent. Relat. Res. 2014, 16, 155-165. [CrossRef]

30. Conserva, E.; Generali, L.; Bandieri, A.; Cavani, F.; Borghi, F.; Consolo, U. Plaque accumulation on titanium disks with different surface treatments: An in vivo investigation. Odontology 2018, 106, 145-153. [CrossRef]

31. Rodríguez Sánchez, F.; Rodríguez Andrés, C.; Arteagoitia, I. Which antibiotic regimen prevents implant failure or infection after dental implant surgery? A systematic review and meta-analysis. J. Cranio-Maxillofac. Surg. 2018, 46, 722-736. [CrossRef]

32. Louropoulou, A.; Slot, D.E.; Van der Weijden, F. The effects of mechanical instruments on contaminated titanium dental implant surfaces: a systematic review. Clin. Oral Implants Res. 2014, 25, 1149-1160. [CrossRef]

33. Ramaglia, L.; di Lauro, A.E.; Morgese, F.; Squillace, A. Profilometric and standard error of the mean analysis of rough implant surfaces treated with different instrumentations. Implant Dent. 2006, 15, 77-82. [CrossRef]

34. Speelman, J.A.; Collaert, B.; Klinge, B. Evaluation of different methods to clean titanium abutments. A scanning electron microscopic study. Clin. Oral Implants Res. 1992, 3, 120-127. [CrossRef]

35. Kiska, D.L.; Gilligan, P.H. Pseudomonas. In Manual of clinical microbiology, 8th ed.; Murray, P.R., Baron, E.J., Jorgensen, J.H., Pfaller, M.A., Yolken, R.H., Eds.; ASM Press: Washington, DC, USA, 2003; pp. 719-728.

36. Albertini, M.; López-Cerero, L.; O’Sullivan, M.G.; Chereguini, C.F.; Ballesta, S.; Ríos, V.; Herrero-Climent, M.; Bullón, P. Assessment of periodontal and opportunistic flora in patients with periimplantitis. Clin. Oral Impl. Res. 2015, 26, 937-941. [CrossRef] 